\title{
$\bullet$ \\ Effect of Lower Limb Exercises on Core Muscles in Functional Activities in Low Back Pain Subjects
}

\section{IJCRR}

Section: Healthcare

ISI Impact Factor

(2019-20): 1.628

IC Value (2019): 90.81

$\operatorname{SJIF}(2020)=7.893$

\section{Kotteeswaran $\mathbf{K}^{1}$, Veluri $\mathbf{R}^{2}$, Neelamegan $\mathbf{A}^{3}$, Shalini $\mathbf{S}^{3}$}

'Professor, Saveetha College of Physiotherapy, Chennai, India; ${ }^{2} \mathrm{MPT}$ (Orthopaedics), Saveetha College of Physiotherapy, Chennai, India; ${ }^{3} \mathrm{MPT}$ (Orthopedics and Manual Therapy) Student, Saveetha College of Physiotherapy, Chennai, India.

\section{ABSTRACT}

Introduction: Low back pain is a common health problem in human beings and about 5 to $15 \%$ will develop low back pain (LBP). The clinical Findings of LBP suggest that lumbar mobility is decreased and the recruitment order of core muscles is altered. In the literature, there is no data about the effect of core muscle strengthening in the chronicity (short duration, long duration) of LBP.

Aim: To find the effectiveness of lower limb exercises on core muscles in subjects with low back pain in reducing pain and improving functional activities.

Methods: 30 patients with low back pain were selected based on the inclusion and exclusion criteria. The subjects were divided into two groups namely Group-A \& Group-B with 15 subjects in each group. Group-A subjects received Lower Limb Exercises and Interferential therapy, Group-B subjects received Traditional low back Exercises and Interferential therapy. The outcome measures are the Numerical pain rating scale (NPRS) and Oswestry disability index (ODI). The statistics of this study all the values were tabulated and statistically analyzed by using paired and unpaired t-test.

Result: Student t-test analysis revealed a significant difference $(p<0.0001)$ between the two groups in the parameter of the Numerical pain rating scale and Oswestry disability index.

Conclusion: This study shows better improvement in improving Core muscles and decreasing pain Lower limb exercises Group A than Traditional low back exercises Group B in low back pain subjects.

Key Words: Core muscle, Lower limb exercises, Traditional low back exercises, Functional activities, Low back pain

\section{INTRODUCTION}

Low back pain is one of the commonest musculoskeletal disorders, affecting up to $90 \%$ of people at some point in their lifetime. Up to $50 \%$ will have more than one episode of back pain. ${ }^{1}$ Low back pain (LBP) is not a specific disease, rather it is a symptom.

Low back pain is a common disorder involving the muscles, nerves, and bones of the back which affects nearly $60-80 \%$ of people throughout their lifetime. The prevalence rate of low back pain is reported to be high as $84 \%$, and studies have quoted that $11-12 \%$ of the population is being disabled by low back pain. A precise cause of mechanical back pain can be identified in only 5-15\% per cent of the time and the exact cause cannot be identified in $85-95 \%$ of the population.
Back pain is widely categorized into 2 types namely mechanical \& non-mechanical. Mechanical pain is the general term that refers to any type of back pain caused by placing abnormal or increased or altered biomechanical stress over the stable spine. The high endurance muscles over the low back and core muscles and ligaments surrounding the spine provide the best protection against low back pain. ${ }^{3}$

The contraction of core muscles associated with the movement of the lower limb has been shown to contribute to the maintenance of the position of the centre of mass over the base of support and the stability of affected joints. ${ }^{4}$ Agreeing with this model, several authors have identified contraction of the rectus abdominal muscle and the erector spinal muscle in advance of upper-limb flexion and extension. ${ }^{5}$ This muscle activity, occurring before or shortly after the onset of activity of the prime mover of the limb, is referred to as feed-

\section{Corresponding Author:}

Dr. K. Kotteeswaran, Professor, Saveetha College of Physiotherapy, Saveetha Institute of Medical and Technical Sciences, Chennai, India. Email:k.kotteeswaran@gmail.com

ISSN: 2231-2196 (Print)

Received: 27.01.2021
ISSN: 0975-5241 (Online)

Revised: 03.03 .2021
Accepted: 12.04 .2021 
forward because it cannot be initiated by feedback from the limb movement. ${ }^{6}$ Recent evidence indicates that the lumbar multifidus muscle $(\mathrm{MF})^{7}$ and transversus abdominis muscle (TrA) may be involved in controlling spinal stability. Importantlyfound that the transversus abdominis muscle contracted before the other abdominal muscles when the trunk was loaded by applying a weight ventrally to a harness over the shoulders. ${ }^{8}$

Studies have evaluated only the feed-forward muscular response to the upper-limb movement. ${ }^{9}$ Due to the anatomical proximity and functional interrelationship between the hip and spine. ${ }^{10}$ However, we evaluated the movement of the lower limb for a more appropriate investigation of the lumbar spine. But it has been studied that the evaluation of lower limb movement in a standing position is complex because the body is required to deal with two distinct challenges to postural equilibrium. The body deals with this challenge by shifting the weight over the other leg before the movement of the limb. ${ }^{11}$

In the 1990s, intensive strengthening exercises were highlighted. ${ }^{12}$ More recently, new evidence demonstrating the effect of the segmental stabilizing exercises has been emerging. The approach of the segmental stabilizing exercise is considerably different from the traditional strengthening exercises. $^{13}$

The Core Strength below can be utilized as a preventative rehabilitation program or if you are recovering from an injury. The program includes a strengthening section that should be done 3-4 times a week. The "core" is comprised of several groups of muscles including the transversus abdominus, multifidus, diaphragm and pelvic floor muscles and their primary function is to work together to produce maximum stability in the abdominal and lumbar back region, as well as coordinate the movement of the limbs and spine. Therefore, it is important to learn how to effectively co-contract these muscles while performing these rehabilitation exercises.

The traditional physiotherapy modalities used for the management of low back pain include Interferential Therapy, Transcutaneous electrical nerve stimulation, short wave diathermy, ultrasound and the exercise program selected for the rectus abdominus and oblique abdominal muscles. Interferential therapy is an electrotherapeutic modality used to treat pain. Interferential Therapy decreases musculoskeletal pain by increasing circulation, promoting efflux of pain-inducing chemicals from the site and bye gate control therapy.

The Oswestry Disability Index (ODI) is an index derived from the Oswestry Low Back Pain Questionnaire used by clinicians and researchers to quantify disability for patients with low back pain. ${ }^{14}$ The self-completed questionnaire consists of components like the intensity of pain, lifting, ability to care for oneself, ability to walk, ability to sit, sexual func- tion, ability to stand, social life, sleep quality and ability to travel. Each question is scored on a 5 point Linkert scale with the first level indicating the least amount of disability and the last level is scored 5 indicating the most severe disability. The scores for all questions answered are summed and multiplied to obtain the index ranging from 0 to 100 .

\section{MATERIALS AND METHODS}

The study was conducted in Saveetha Medical college, Physiotherapy outpatient department, with study design as a Quasi-Experimental study after obtaining approval from the Institutional ethical committee (029/02/2017/IEC/SU). Sampling method as systematic random sampling and a sample size of 30 subjects divided into two groups with 15 subjects in each group. subjects were included in the study based on the inclusion criteria as Both males and females are diagnosing back pain with an age of 18 to 40 years and subjects were excluded from the study if there was any (a) History spinal surgeries, (b) Deformities of spine and lower limb pathology, (c) Tuberculosis (TB) spine patients, (d) If they were treated due to other neurological problems, (e) They had acute inflammation, tumours and (f) if there were pregnant.

\section{PROCEDURE}

According to the inclusion and exclusion criteria before the study, the principal researchers explained the procedure to all the subjects and informed consent was obtained, using systemic random sampling30 subjects will be selected from Physiotherapy - outpatient department and will be allocated into two groups namely control and experimental group 15 subjects in each group. After recruitment on eligibility, informed consent will be obtained on explaining the treatment to be given. Group A experimental group - is treated with lower limb exercises combines with interferential therapy and it is termed as the experimental group. Group B control group is treated with traditional low back exercises combined with interferential therapy. The pain was measured on the Numerical pain rating scale (NPRS) and low back pain disability was assessed by the Oswestry disability index (ODI). The values were considered as the pre-test. After 4 weeks of treatment again pain is measured using the Numerical pain rating scale (NPRS) and low back pain disability is assessed using the Oswestry disability index (ODI) and the values obtained were considered as post-test values.

Group-A: Lower Limb Exercises 15 subjects were included in this group and each subject was positioned on the treatment table. The interventional exercises given to this group are Bilateral straight leg raising, Dead bug, Prone flutters, Bird dog using $1 \mathrm{~kg}$ weight cuffs, Pelvic bridging, Side plank and Knee hug. 


\section{A.) Bilateral straight leg- raising:}

Patient position: Patient is supine lying with the hips at 50 degrees and knees extended using with $1 \mathrm{Kg}$ weight cuff on both sides and lift leg bilateral.

\section{B.) Bird Dog}

Bird Dogs are an excellent way to strengthen multiple muscles in the core. They not only target the abdominals but also challenge erector spinae along the spine, glutes.

Patient position: Activate your stomach muscles by getting onto your hands and knees so your low back flattens like a tabletop. Without allowing your pelvis to tilt, lift the opposite arm and leg in the air with a $1 \mathrm{~kg}$ weight cuff until each is fully extended. hold the position for 5 to 10 seconds and repeat with the opposite limbs.

\section{C.) Dead Bug}

This exercise targets the abdominal muscles to maintain core stability.

Patient position: While lying on your back, lift both legs in the air and bend your hips and knees to 90-degree. Squeeze your stomach muscles and instruct patients to keep their backs flat against the ground. Now gradually straighten one leg in the air using with $1 \mathrm{~kg}$ weight cuff as you raise the alternate arm overhead. Then repeat with the opposite arm and leg.

\section{D.) Prone Flutters}

This exercise challenges the multifidus muscles in the low back which is referred to as the primary stabilizer of the spine.

Patient position: Lie on your stomach with arms extended overhead. Lift right arm and left leg simultaneously in the air and use with $1 \mathrm{~kg}$ weight cuff. Repeat with the opposite arm and leg.

\section{E.) Pelvic bridging}

Bridges activate several different core muscles including your gluteus maximus and transversus abdominus.

Patient position: The patient is in the hook-lying position, have the patient press the upper back and feet into the mat, elevate the pelvis, and extend the hips.

\section{F.) Side Plank}

Side planks target the oblique muscles and the gluteus Medius, an important core muscle on the side of the pelvis.

Patient position: Lie on the right side with knees straight and legs stacked on top of each other. With the right elbow positioned under the shoulder, lift the body off the ground until the spine is straight and hold this position for 5 to $10 \mathrm{sec}-$ onds. Repeat the exercise on the left side.

\section{G.) Knee hug}

Patient position: Lie on back with legs extended and back straight. Keep hips level and lower down on the floor. Bend both knees and hugs the knees towards the chest, placing hands on the back of the thigh.

GROUP-B: Traditional low back exercises: 15 subjects were included in this group and each subject was positioned on the treatment table. The interventional exercises given to this group are traditional low back exercises.

These exercises are done and training up to four weeks with 10 seconds (Hold \& Relax) 10 repetitions maximum of each exercise, 3 sessions a week for four weeks. Interferential therapy patients of both groups were administered interferential therapy for 3 sessions a week for four weeks. The duration of the treatment is 15 minutes. The placement of the electrode is in the low back region.

Outcomes measures are Numerical pain rating scale (NPRS) and Oswestry disability index (ODI) was taken before the intervention and after the end of the $4^{\text {th }}$ week and considered as pre \& post values. Statistically, results were obtained.

\section{STATISTICAL ANALYSIS}

The collected data was analyzed using descriptive and inferential statistics. To all parameters mean and standard deviation (SD) were used. Paired t-test was used to analyze significant changes between pre-test $\&$ post-test measurements. An unpaired t-test was used to analyze significant changes between the two groups.

\section{RESULTS}

The statistical analysis made with the quantitative data revealed a significant difference between pre-test and post-test values of the Numerical Pain Rating Scale (NPRS) and Oswestry disability index (ODI)

\section{Pre-test and Post-test values of Group A}

The pre-test mean value of NPRS is 8.20 with a standard deviation of 0.86 and the post-test mean value of NPRS is 2.40 with a standard deviation of 0.51 , this shows that NPRS scores are gradually increasing with the P-value $(<0.0001)$ statistically significant.

The pre-test mean value of ODI is 76.67 with a standard deviation of 18.01 and the post-test mean value of ODI is 18.53 with a standard deviation of 1.77 , this shows that ODI scores are gradually increasing with the p-value $(<0.0001)$.

\section{Pre-test and Post-test values of Group B}

The pre-test mean value of NPRS is 8.20 with a standard deviation of 0.86 and the post-test mean value of NPRS is 
3.60 with a standard deviation of 0.83 , this shows that NPRS scores are gradually increasing with the p-value $(<0.0001)$ statistically significant.

The pre-test mean value of ODI is 82.40 with a standard deviation of 15.18 and the post-test mean value of ODI is 28.40 with a standard deviation of 6.47 , this shows that ODI scores are gradually increasing with the P-value (0.0001).

\section{Comparison between NPRS and ODI for Groups} $A$ and $B$

The post-test mean value of the Numerical pain rating scale in Group-A is 2.40 with 0.51 and the post-test mean value of the Numerical pain rating scale in Group-B is 3.60 with 0.83 , this shows that Group-A is greater than Group-B with the p-value $(<0.0001)$.

The post-test mean value of Oswestry disability index in Group-A is 18.53 with 1.77 and the post-test mean value of Oswestry disability index in Group-B is 28.40 with 6.47, this shows that Group-A is greater than Group-B with the P-value $(<0.0001)$.

\section{DISCUSSION}

Chronic low back pain (LBP) is a serious global public health problem, which is considered to be the leading cause of absence from work and limitation of activity all over the world. ${ }^{15}$ The present study was conducted to study the effectiveness of Lower limb exercises combined with Interferential Therapy in the treatment of low back pain by comparing with Traditional low back exercises combined with Interferential therapy. Both the treatment was done for 3 days per week for four weeks. A numerical pain rating scale and Oswestry low back pain disability index were used to measure the outcome.

The current study rigorously tested Lower limb exercises to determine whether this exercise is effective in low back pain. The study results showed statistically and clinically significantly reducing low back pain. The stability of the spine is given by abdominal muscles. Therefore, rehabilitation programs including low back exercises concentrating on strengthening the abdominal muscles combined with core stability and proprioception will reduce the risk of low back pain when performed regularly.

The basic physiology behind the importance of core muscles strength followed by the exercises might be the postural mechanism. ${ }^{5}$ In the normal scenario when the lower limb muscles are moving the core muscle stabilizers have to stabilize to the vertebral column particularly the lower lumbar spine and pelvis. Chari VR in 1986 stated that the EMG activity of back muscle continuity firing until the lower limb movement took place, which clearly states when the lower limb exercises are performed the core muscles which are predominantly stabilizers, will always contract. Hence it is assumed that lower limb exercises will contribute to core muscle strength.

In theory, stated by Son. K et al in 1988 the trunk, hip, knee, and ankle joints are postural set muscles that always contracted together to maintain posture. The activation of one group of muscle result co-activation of all the other group of muscles. Going by this concept the study hypothesizes have been strongly substantiated.

The mechanical benefit of lower limb exercises to strengthen core muscles is that subjects who have severe pain on spinal movement can start performing the lower limb exercises which invariably result in the strengthening of the core muscles. These also result in acceleration of the rehabilitation process, where the physiotherapists have the liberty of teaching exercises from day one irrespective of pain.

The patient had difficulty holding period exercises. The patient who is obese suffered from breathing difficulties during the exercises period. Even some of the risk factors like diabetes also influenced the patient during exercises where fatigue is concerned. They were difficulty in doing some of the exercises which resulted in increasing the pain of low back.

The Core Strength below can be utilized as a preventative rehabilitation program or if you are recovering from an injury. The program includes a strengthening section that should be done 3-4 times a week. The transversus abdominus, multifidus, diaphragm and pelvic floor muscles work together to produce maximum stability in the abdominal and lumbar back region, as well as coordinate the movement of the arms, legs, and spine. Hence, it is important to learn to effectively cocontract these muscles while performing rehabilitation. The traditional physiotherapy modalities used for the management of low back pain include IFT and the traditional low back exercise program selected for the rectus abdominus and oblique abdominal muscles. A Systematic Review and Meta-analysis concluded that Interferential Current Therapy is having a beneficial effect in the management of musculoskeletal pain. ${ }^{16}$

So, this study is intended to find out the effects of lower limb exercises to core muscles in functional activities in low back pain. This study showed that Lower limb exercises had additional treatment effects compared with the control group.

\section{CONCLUSION}

This study shows better improvement in improving Core muscles and decreasing pain Lower limb exercises Group A than General low back exercises Group B in low back pain subjects. Both the treatments i.e., Lower limb exercises and General Low back exercises are almost equal in their clinical effectiveness for improving core muscles. 


\section{ACKNOWLEDGEMENTS}

The author acknowledges the support received from the staff at the Department of Physiotherapy, Saveetha University, Chennai. The author also acknowledges his colleagues for their assistance in recruiting participants for the study and finally acknowledge all the subjects who took part in the study.

\section{Conflict of Interest: Nil}

Source of Funding: Self

\section{REFERENCES}

1. Arya RK. Low back pain -signs, symptoms, and management.J Int Ass. Curr Med. 2014;15(1):30-41.

2. Leon Chaitow. muscle Energy Techniques. 2010. 2nd edition, 44-55.

3. Venu Akuthota, Scott F. Nadler. Core strengthening. Arch Phys Med Rehab. 2004;85(3):86-92.

4. Bouisset S, Zattara M. A sequence of postural adjustments precedes voluntary movement. Neurosci Lett.1981;22:263-270.

5. Friedli WG, Hallet M, Simon SR. Postural adjustments associated with rapid voluntary arm movements. Journal of neurology, neurosurgery and psychiatry. 1984;47:611-622.

6. Aruin AS, Latash ML. Directional specificity of postural muscles in feed-forward postural reactions during fast voluntary arm movements. Exp Brain Res. 1995; 103:323-332.

7. Wilke HJ, Wolf S, Claes LE. Stability increase of the lumbar spine with different muscle groups: a biomechanical in vitro study.Spine. 1995; 20:192-198.
8. Cresswell AG, Ckundstrom H, Thorstensson A. Observations on intra-abdominal pressure and patterns of abdominal intra-muscular activity in man. Acta Physiol Scand.1992;144:409-418.

9. Belen'kii V, Gurfinkel VS, Paltsev Y. Elements of control of voluntary movements. Bzojzika. 1967; 12: 135-141.

10. Paquet N, Malouin F, Richards CL. Hip-spine movement interaction and muscle activation patterns during sagittal trunk movements in low back pain patients. Spine.1994;19:596-603.

11. Mouchnino L, Autrey R, Massion J, Pedotti A. Coordination between equilibrium and head-trunk orientation during leg movement: a new strategy built up by training. J Neurophysiol.1992;67:1587-1598.

12. Carpenter DM, Nelson BW. Low back strengthening for the prevention and treatment of low back pain. Med sci sports Exerc. 1999;31:18-24.

13. Richardson A, Snjider C. The Relation Between the Transversus Abdominus Muscles, Sacroiliac Joint Mechanism and low back pain. Spine. 2002; 27: 399-405.

14. Fairbank JCT, Couper J, Davies JB, O'Brian JP. The Oswestry low back pain disability questionnaire. Physiotherapy. 1980; 66:271-3.

15. Deepali sheth, James Ghagare. Variation in beliefs towards low back pain between physiotherapy and nursing students. Int J Curr Res Rev. 2018;10(9):11-14.

16. Jorge P. Fuentes, Susan Armijo Olivo, David J M Agee, Douglas P. Gross. Effectiveness of Interferential Current Therapy in the management of musculoskeletal pain: A Systematic Review and meta-analysis. Phys Ther. 2010;90(9):1219-38.

Table 1: Pre-test - Post-test values of Group A NPRS and ODI

\begin{tabular}{llccc} 
GROUP A & & Mean & Standard deviation & 'p' value \\
NPRS & Pre-test & 8.20 & 0.86 & $<0.0001$ \\
& Post-test & 2.40 & 0.51 & \\
ODI & Pre-test & 76.67 & 18.01 & $<0.0001$ \\
& Post-test & 18.53 & 1.77 & \\
\hline
\end{tabular}

Table 2: Pre-test - Post-test values of Group B NPRS and ODI

\begin{tabular}{llccc} 
GROUP A & & Mean & Standard deviation & p -value \\
\multirow{2}{*}{ NPRS } & Pre-test & 8.20 & 0.86 & $<0.0001$ \\
& Post-test & 3.60 & 0.83 & \\
\multirow{2}{*}{ ODI } & Pre-test & 82.40 & 15.18 & $<0.0001$ \\
& Post-test & 28.40 & 6.47 & $<$ \\
\hline
\end{tabular}

Table 3: Post-test values of Group A and Group B NPRS and ODI

\begin{tabular}{lccccc} 
Parameter & \multicolumn{3}{c}{ Post-test values } & Group B \\
& \multicolumn{2}{c}{ Group A } & \multicolumn{2}{c}{ p-value } \\
NPRS & 2.40 & 0.51 & 3.60 & 0.83 & $<0.0001$ \\
ODI & 18.53 & 1.77 & 28.40 & 6.47 & $<0.0001$ \\
\hline
\end{tabular}

